TRANSACTIONS OF THE

AMERICAN MATHEMATICAL SOCIETY

Volume 353, Number 7, Pages 2705-2724

S 0002-9947(01)02692-7

Article electronically published on March 12, 2001

\title{
PRIMES IN SHORT ARITHMETIC PROGRESSIONS WITH RAPIDLY INCREASING DIFFERENCES
}

\author{
P. D. T. A. ELLIOTT
}

\begin{abstract}
Primes are, on average, well distributed in short segments of arithmetic progressions, even if the associated moduli grow rapidly.
\end{abstract}

\section{Statement of Results}

In this paper I establish two results concerning the distribution of prime numbers in short segments of residue classes to widely separated moduli.

Let $\Lambda(n)$ denote von Mangoldt's function, $\log p$ if $n$ is a power of a prime $p$, zero otherwise. For integers $D>0$ and $r$, let $\psi(x, D, r)$ denote the sum of the $\Lambda(n)$ over the positive integers not exceeding $x$ which lie in the residue class $r(\bmod D)$.

Let $f$ be a polynomial of $\operatorname{deg}($ ree $)$ at least one, with integer coefficients, leading coefficient positive.

Theorem 1. If $A>0,4 \beta \operatorname{deg} f<1$, then

$$
\sum_{D \leq x^{\beta}} \frac{\phi(f(D))}{D} \max _{(r, f(D))=1} \max _{y \leq x}\left|\psi(y, f(D), r)-\frac{y}{\phi(f(D))}\right| \ll x(\log x)^{-A},
$$

the summation confined to integers $D$ for which the modulus $f(D)$ is positive.

In a recent paper, [5, investigating the solution of polynomial equations in primes, I employ a lower bound for a doublesum

$$
\sum_{\substack{p+1=m f(q), x / 2<m \leq x \\ x^{\alpha} / 2<q \leq x^{\alpha}}} r(m)
$$

the sum taken over representations $m=(p+1) f(q)^{-1}$ of integers $m$ by primes $p, q$. My appeal in that paper to elaborate results of Linnik, Fogels, Gallagher and others may be replaced by the application of Theorem 1 . In the notation of that paper, the lower bound improves to an asymptotic estimate

$$
\left(1+O\left(\frac{1}{(\log x)}\right)\right) \frac{\phi(\Delta) P x^{1+\alpha}}{4 \alpha(1+\alpha \operatorname{deg} f) \Delta \phi(P)(\log x)^{2}},
$$

valid for each positive $\alpha$ not exceeding the $\beta$ of Theorem 1 .

Received by the editors January 7, 1999 and, in revised form, February 26, 2000.

2000 Mathematics Subject Classification. Primary 11N13; Secondary 11B25.

Partially supported by NSF contract DMS-9530690. 
When $f$ is a linear polynomial, the Bombieri-Vinogradov theorem guarantees the estimate of Theorem 1 for any $\beta<1 / 2$ [3]. Under the tighter restriction $4 \beta \operatorname{deg} f<1$ a bound

$$
\sum_{\frac{1}{2} x^{\beta}<D \leq x^{\beta}} \max _{(r, f(D))=1} \max _{y \leq x}\left|\psi(y, f(D), r)-\frac{y}{\phi(f(D))}\right| \ll x(\log x)^{-A}
$$

in the case of a polynomial of degree 2 or more is immediate, but it is considerably weaker than the estimate $\ll x^{1+\beta-\beta \operatorname{deg} f}$ that follows from the wellknown Brun-Titchmarsh theorem. Theorem 1 delivers the more appropriate $\ll$ $x^{1+\beta-\beta \operatorname{deg} f}(\log x)^{-A}$.

The following result will illustrate the extent to which I employ the polynomial nature of the moduli $f(D)$.

Theorem 2. Let $\delta>0, A>0$. Then

$$
\begin{aligned}
& \sum_{j=1}^{J} \max _{\left(r, s D_{j}\right)=1} \max _{y \leq x}\left|\psi\left(y, s D_{j}, r\right)-\frac{\psi(y, s, r)}{\phi\left(D_{j}\right)}\right| \\
& \ll \frac{x}{\phi(s)(\log x)^{A}} \sum_{j=1}^{J} \frac{1}{\phi\left(D_{j}\right)}+\frac{x}{s}\left(\sum_{\substack{u=1 \\
\left(D_{u}, D_{v}\right)>1}}^{J} \sum_{\substack{v \\
D_{u} D_{v}}}^{J}\right)^{1 / 2}(\log x)^{4200}
\end{aligned}
$$

for all $x \geq 2$ and positive integers $s, D_{j}$ satisfying $s D_{j} \leq x^{\frac{1}{4}-\delta}, 1 \leq s \leq x^{\delta}$, and $\left(s, D_{j}\right)=1, j=1, \ldots, J$.

The general argument is a largely self-contained careful application of Linnik's Dispersion Method, without appeal to Fourier Analysis on $\mathbb{R} / \mathbb{Z}$.

I conclude the paper with a discussion of interesting problems that arise in the pursuit of wider uniformities in Theorems 1 and 2.

\section{INTRODUCTION OF MULTILINEAR FORMS}

For a positive integer $k$ and complex number $s$ define

$$
M(s)=\sum_{n \leq x^{1 / k}} \mu(n) n^{-s}
$$

The following identity is clear (Heath-Brown [9], cf. Linnik [11, Introduction, pp. 21-22).

\section{Lemma 1.}

$$
\frac{\zeta^{\prime}(s)}{\zeta(s)}=\sum_{j=1}^{k}(-1)^{j-1}\left(\begin{array}{c}
k \\
j
\end{array}\right) \zeta(s)^{j-1} \zeta^{\prime}(s) M(s)^{j}+\frac{\zeta^{\prime}(s)}{\zeta(s)}(1-\zeta(s) M(s))^{k}
$$

whenever $\operatorname{Re}(s)>1$.

Since the Dirichlet series $(1-\zeta(s) M(s))^{k}$ has no terms $a_{n} n^{-s}$ with $n \leq x$, for any arithmetic function $g$ whatsoever,

$$
\sum_{\substack{n \leq x \\
n \equiv b(\bmod D)}} g(n) \Lambda(n)=\sum_{r=1}^{k}(-1)^{r-1}\left(\begin{array}{l}
k \\
r
\end{array}\right) S_{r}
$$


where

$$
S_{r}=\sum_{\substack{n_{1} \cdots n_{r} m_{1} \cdots m_{r} \leq x \\ n_{1} \cdots n_{r} m_{1} \cdots m_{r} \equiv b(\bmod D)}} \cdots\left(n_{1} \cdots n_{r} m_{1} \cdots m_{r}\right) \log n_{1} \mu\left(m_{1}\right) \cdots \mu\left(m_{r}\right) .
$$

The treatment of a typical multilinear form differs according to the size of (the product) $n_{1} \cdots n_{j}$. We first assume it large.

\section{Divisor FUnCTIONS ON ARITHMETIC PROGRESSIONS}

Although the approach is classical, pioneered by Linnik [10, I tailor the results to the situation at hand.

For the duration of this section $L(s, \chi)$ will denote the series $\sum_{n=1}^{\infty} \chi(n) n^{-s}$ formed with the Dirichlet character $\chi$ defined on the multiplicative group of reduced residue classes $(\bmod D), D \geq 1$.

\section{Lemma 2.}

$$
\sum_{\chi(\bmod D)} \int_{-T}^{T}\left|\sum a_{n} \chi(n) n^{i t}\right|^{2} d t \ll \sum\left|a_{n}\right|^{2}(n+T \phi(D))
$$

uniformly for $D \geq 1, T>0$ and complex $a_{n}$ for which the series $\sum\left|a_{n}\right|^{2} n$ converges.

Proof. It will suffice to establish the inequality for an arbitrary finite collection of complex numbers $a_{n}$. With $\tau=e^{1 / T}$,

$$
\int_{-T}^{T}\left|\sum a_{n} n^{i t}\right|^{2} d t \ll T^{2} \int_{0}^{\infty}\left|\sum_{y<n \leq y \tau} a_{n}\right|^{2} \frac{d y}{y},
$$

Gallagher [8].

From the orthogonality of Dirichlet characters $(\bmod D)$,

$$
\sum_{\chi(\bmod D)}\left|\sum_{u<n \leq v} a_{n} \chi(n)\right|^{2} \ll(v-u+\phi(D)) \sum_{u<n \leq v}\left|a_{n}\right|^{2},
$$

treating the cases $v-u<1, v-u \geq 1$ separately. The sum to be estimated in the lemma is then

$$
\begin{aligned}
& \ll T^{2} \int_{0}^{\infty} \sum_{y<n \leq y \tau}\left|a_{n}\right|^{2}((\tau-1) y+\phi(D)) \frac{d y}{y} \\
& \ll T^{2} \sum\left|a_{n}\right|^{2} \int_{n / \tau}^{n}((\tau-1) y+\phi(D)) \frac{d y}{y} \ll \sum\left|a_{n}\right|^{2}(n+T \phi(D)) .
\end{aligned}
$$

\section{Lemma 3.}

$$
\sum_{\chi \neq \chi_{0}(\bmod D)} \int_{-T}^{T}|L(\sigma+i t, \chi)|^{4} d t \ll \phi(D)(T+2)(\log D(T+2))^{6}
$$

uniformly for $|\sigma-1 / 2| \leq 2(\log D(T+2))^{-1}$.

The power of the logarithm may be reduced and the principal character $\chi_{0}$ included in the summation, but we shall not need these refinements. 
Proof. Since

$$
L(s, \chi)^{2}=\sum_{n=1}^{\infty} \tau(n) \chi(n) n^{-s}, \quad \operatorname{Re}(s)>1,
$$

we may interchange integration and summation to obtain

$$
\frac{1}{2 \pi i} \int_{c-i \infty}^{c+i \infty} L(s+z, \chi)^{2} \Gamma(z) Y^{z} d z=\sum_{n=1}^{\infty} \tau(n) \chi(n) n^{-s} e^{-n / Y}
$$

for real $Y>0, c>0$. For non-principal characters both sides are analytic and so equal in the whole complex $s$-plane.

Moving the integral to the line $\operatorname{Re}(z)=c$ with $-1<c<0$, we pass over the simple pole of $\Gamma(z)$ at $z=0$ which gives to the integrand a residue $L(s, \chi)^{2}$.

Let $R=D(2+T)$. In terms of the functional equation

$$
L(s, \chi)=\psi(s, \chi) L(1-s, \bar{\chi})
$$

we may decompose the (new) integral as

$$
\begin{aligned}
& \frac{1}{2 \pi i} \int_{d-i \infty}^{d+i \infty} \psi(s+z, \chi)^{2} \sum_{n \leq R} \tau(n) \bar{\chi}(n) n^{-(1-s-z)} \Gamma(z) Y^{z} d z \\
& \quad+\frac{1}{2 \pi i} \int_{c-i \infty}^{c+i \infty} \psi(x+z, \chi)^{2} \sum_{n>R} \tau(n) \bar{\chi}(n) n^{-(1-s-z)} \Gamma(z) Y^{z} d z
\end{aligned}
$$

with $d$, initially the same as $c$, permitted any value in the interval $(-1,0)$.

Set $c=-1 / 2-3(\log R)^{-1}, d=-(\log R)^{-1}, Y=R$.

If $\chi$ is induced by the primitive character $\chi_{1}\left(\bmod D_{1}\right), D_{1} \mid D$, then

$$
L(s, \chi)=L\left(s, \chi_{1}\right) \prod_{p \mid D}\left(1-\frac{\chi_{1}(p)}{p^{s}}\right)
$$

so that $\psi(s, \chi)$ becomes

$$
\psi\left(s, \chi_{1}\right) \prod_{p \mid D}\left(1-\frac{\chi_{1}(p)}{p^{s}}\right)\left(1-\frac{\overline{\chi_{1}(p)}}{p^{1-s}}\right)^{-1} .
$$

For $3 / 8 \leq \operatorname{Re}(s) \leq 5 / 8$ the product over the divisors of $D$ has a representation

$$
\exp \left(\sum_{p \mid D}\left\{-\frac{\chi_{1}(p)}{p^{s}}+\frac{\bar{\chi}_{1}(p)}{p^{1-s}}+\frac{\chi_{1}(p)^{2}}{2 p^{2 s}}-\frac{\bar{\chi}_{1}(p)^{2}}{2 p^{2(1-s)}}+O(1)\right\}\right) \text {. }
$$

In the band $|\operatorname{Re}(s)-1 / 2| \leq 2(\log R)^{-1}, p^{-(1-s)}-p^{-\bar{s}} \ll p^{-1 / 2}\left(\exp \left(\frac{4 \log p}{\log D}\right)-1\right)$. The real part of the sum over the prime divisors of $D$ in the exponential is

$$
\ll \sum_{p \mid D} \frac{\log p}{p^{1 / 2} \log D} \ll 1 .
$$

On $\operatorname{Re}(z)=d$ we obtain $\psi(s+z, \chi) \ll(2+|z|)^{1 / \log R}$, cf. Prachar [13], VII, Satz 1.1, p. 207 and Anhang Satz 6.2, p. 395, hence $\psi(x+z, \chi) \ll(2+|z|)^{2}$ for $s$ in the narrow strip about $\operatorname{Re}(s)=1 / 2$. 
On $\operatorname{Re}(z)=c$ we obtain $\psi\left(s+z, \chi_{1}\right) \ll D_{1}^{1 / 2}(T+2+|z|)^{1 / 2+5 / \log R}$. The modifying product does not exceed $c_{1}^{\nu\left(D / D_{1}\right)}$ for a certain positive $c_{1}$, and so is $\ll\left(D / D_{1}\right)^{1 / 2}$. Hence $\psi(s+z, \chi) \ll R^{1 / 2}(2+|z|)^{11}$ for $s$ in the narrow strip.

Thus

$$
\begin{aligned}
L(s, \chi)^{2} \ll & \left|\sum_{n=1}^{\infty} \tau(n) \chi(n) n^{-s} e^{-n / R}\right| \\
& +\log R \int_{d-i \infty}^{d+i \infty}\left|\sum_{n \leq R} \tau(n) \bar{\chi}(n) n^{-(1-s-z)}\right||\Gamma(z+1)|(2+|z|)^{4} d \operatorname{Im}(z) \\
& +R^{1 / 2} \int_{c-i \infty}^{c+i \infty}\left|\sum_{n>R} \tau(n) \bar{\chi}(n) n^{-(1-s-z)}\right||\Gamma(z+1)|(2+|z|)^{22} d \operatorname{Im}(z)
\end{aligned}
$$

uniformly for $|\operatorname{Re}(s)-1 / 2| \leq 2(\log R)^{-1}$.

Since

$$
\int_{h-i \infty}^{h+i \infty}|\Gamma(z+1)|(2+|z|)^{22} d \operatorname{Im}(z)
$$

is bounded uniformly for $-1<h<0$, applications of the Cauchy-Schwarz inequality and the previous lemma complete the proof. For example, on $\operatorname{Re}(z)=d$ with $s=\sigma+i t$

$$
\begin{aligned}
& \sum_{\chi \neq \chi_{0}(\bmod D)} \int_{-T}^{T}\left|\sum_{n \leq R} \tau(n) \chi(n) n^{-(1-z-\sigma)+i t}\right|^{2} d t \\
& \ll \sum_{n \leq R} \tau(n)^{2} n^{-1}(n+T \phi(D)) \ll \phi(D)(T+2)(\log R)^{4} .
\end{aligned}
$$

The factor $\log R$ in the corresponding term bounding $L(s, \chi)^{2}$ is wastefully large.

Since $|L(s, \chi)|^{r} \leq 1+|L(s, \chi)|^{4}$ for $0<r<4$, the upper bound of Lemma 3 remains valid with any positive power of $|L(\sigma+i t, \chi)|$ up to the fourth.

\section{Lemma 4.}

$$
\sum_{\chi \neq \chi_{0}(\bmod D)} \int_{-T}^{T}\left|L(1 / 2+i t, \chi)^{r} L(1 / 2+i t, \chi)^{\prime}\right| d t \ll \phi(D)(T+2)(\log D(T+2))^{7}
$$

for $r=1,2$ and 3 .

Proof. With $R$ again $D(T+2)$, Cauchy's integral formula gives

$$
L(s, \chi)^{r} L(s, \chi)^{\prime}=\frac{1}{r+1}\left(L(s, \chi)^{r+1}\right)^{\prime}=\frac{1}{2 \pi i(r+1)} \int \frac{L(w, \chi)^{r+1}}{(w-s)^{2}} d w,
$$

the integral taken over the disc $|w-s|=(\log R)^{-1}$. Taking absolute values we integrate over $t$ and change the order of the two integrations. We see that the expression to be estimated is

$$
\ll \log R \int_{0}^{2 \pi} \sum_{\chi} \int_{-T}^{T}\left|L\left(\sigma+i t+e^{i \theta}(\log R)^{-1}, \chi\right)\right|^{r} d t d \theta
$$


where $\left|\sigma+(\log R)^{-1} \cos \theta-1 / 2\right| \leq 2(\log R)^{-1}$ so that we may apply the previous lemma.

Versions of Lemmas 3 and 4 may be derived from Lemma 10.5 of Montgomery 12. I have adapted (with minor corrections) the more nearly self-contained argument, due to Ramachandra, that is presented in $\S 10$ of Bombieri [3].

Lemma 5. let $0<\alpha<1 / 2, k$ a positive integer. There is a number $\beta$, depending at most upon $k$, so that

$$
\sum_{x_{1}<D m+\ell \leq x} \tau(D m+\ell)^{k} \ll D^{-1}\left(x-x_{1}\right)(\log x)^{\beta}
$$

uniformly for $(\ell, D)=1, D \leq x^{1-\alpha}, x_{1}<x, x-x_{1} \geq x^{1-\alpha / 2}$.

For $x_{1}=0$ the upper bound may be replaced by $x D^{-1}\left(D^{-1} \phi(D) \log x\right)^{2^{k}-1}$.

Proof. See Linnik [11], Lemma 1.1.5 and Lemma 1.1.4. Variant proofs will be indicated following Lemma 14 of the present paper.

Lemma 6. Let $A>0, \delta>0$. Then

$$
\sum_{\substack{n_{1} \cdots n_{r} \leq x \\ n_{1} \cdots n_{r} \equiv b(\bmod D)}} \log n_{1}-\frac{1}{\phi(D)} \sum_{\substack{n_{1} \cdots n_{r} \leq x \\\left(n_{1} \cdots n_{r}, D\right)=1}} \log n_{1} \ll \frac{x(\log x)^{-A}}{\phi(D)}+x^{1 / 2}(\log x)^{8}
$$

uniformly for $(b, D)=1,1 \leq D \leq x^{1-\delta}, x \geq 2, r=1,2,3$ or 4 .

Proof. Without loss of generality we may assume $x$ not to be an integer. The expression, $S(x)$, to be estimated has a representation

$$
\frac{1}{\phi(D)} \sum_{\chi \neq \chi_{0}(\bmod D)} \frac{\bar{\chi}(b)}{2 \pi i} \int_{c-i \infty}^{c+i \infty} L(s, \chi)^{r-1} L(s, \chi)^{\prime} \frac{x^{s}}{s} d s
$$

where the integral may be taken over any line $\operatorname{Re}(s)=c>1$.

Let $B>0$. Replace $x$ by $y$ and average with respect to $y$ over the interval $\left(x-(x \log x)^{-B}, x\right]$. Since $\tau_{r}(n) \leq \tau(n)^{r-1}$, the previous lemma shows that typically

$$
S(x)-S(y) \ll \frac{x}{D}(\log x)^{c_{0}-B},
$$

the constant $c_{0}$ absolute, the implied constant depending upon $B$ and $\delta$. Within a similar error we may replace $x^{s} s^{-1}$ in the integral by the kernel

$$
K(s)=\frac{(\log x)^{B}}{s x} \int_{x-x(\log x)^{-B}}^{x} y^{s} d y .
$$

Of its properties we need only that it is $\ll \min \left(|s|^{-1} x^{\sigma},|s(s+1)|^{-1} x^{\sigma}(\log x)^{B}\right)$ uniformly for $\sigma=\operatorname{Re}(s)>0$.

We move the contour $\operatorname{Re}(s)=c$ to $\operatorname{Re}(s)=1 / 2$. This may be justified by replacing $L(s, \chi)$ with its approximant

$$
\sum_{n \leq Z} \chi(n) n^{-s}+O\left(|s| Z^{-\sigma} D^{1 / 2} \log 2 D\right)
$$

valid in $\sigma=\operatorname{Re}(s) \geq 1 / 4$, likewise replacing $L(s, \chi)^{\prime}$, and letting $Z$ become large. 
Appeal to Lemma 4 shows that the range $|t| \leq x$ contributes

$$
\begin{aligned}
& \ll \frac{1}{\phi(D)} \sum_{\chi \neq \chi_{0}(\bmod D)} \int_{-1}^{1}\left|L(s, \chi)^{r-1} L(s, \chi)^{\prime}\right| \frac{x^{1 / 2}}{|s|} d t \\
& +\sum_{0 \leq k \leq \frac{\log 2 x}{\log 2}} \frac{1}{\phi(D)} \sum_{\chi \neq \chi_{0}(\bmod D)} 2^{-k} \int_{-2^{k}}^{2^{k}}\left|L(s, \chi)^{r-1} L(s, \chi)^{\prime}\right| x^{1 / 2} d t
\end{aligned}
$$

which is $\ll x^{1 / 2}(\log x)^{8}$. For the range $|t|>x$ we argue similarly, employing the second of the bounds on $K(s)$, and obtain a much smaller contribution.

Choosing $B=A+c_{0}$ completes the proof.

The error term in Lemma 6 is $\ll x\left(\phi(D)(\log x)^{A}\right)^{-1}$ provided that $D \leq$ $x^{1 / 2}(\log x)^{-A-8}$, and this will be satisfactory.

A result of the same form as Lemma 6 but with a weaker error term may be obtained for any fixed integer $r$, even by purely elementary means. However, if side conditions are to be placed upon the individual variables $n_{j}, 1 \leq j \leq r$, particularly by introducing factors $n_{j}^{-s_{j}}$ with new complex variables $s_{j}$, then the foregoing argument is altogether more flexible.

When the product $n_{1} \cdots n_{r}$ in a multilinear form of $\S 2$ is small, we may expect the complementary product $m_{1} \cdots m_{r}$ to be large. Since each variable $m_{j}$ is constrained to be small, we may then partition variables and reduce ourselves from a multilinear to a bilinear form.

\section{BILINEAR FORMS ON RESIDUE CLASSES}

Let $s$ be a positive integer, and $D_{j}, 1 \leq j \leq J$, further positive integers, coprime to $s$.

Let

$$
\Delta=\sum_{j}\left|\sum_{m n \equiv u_{j}\left(\bmod D_{j} s\right)} a_{m} b_{n}-\frac{1}{D_{j}} \sum_{\substack{m n \equiv u_{j}(\bmod s) \\\left(n, D_{j}\right)=1}} a_{m} b_{n}\right|
$$

where $\left(u_{j}, D_{j} s\right)=1$ for each $j$, the $a_{m}, b_{n}$ are real numbers supported on $(M / 2, M]$, $(N / 2, N]$ respectively. Note that the second doublesum between the solidi has summation condition $\left(n, D_{j}\right)=1$ rather than $\left(m n, D_{j}\right)=1$, and weight $D_{j}^{-1}$ rather than $\phi\left(D_{j}\right)^{-1}$. I shall assume that $M \geq 2, N \geq 2$

Lemma 7. Let $0<\delta<1,1 \leq s \leq N^{1-\delta}$, and assume that $\left|b_{n}\right| \leq(\tau(n) \log 2 n)^{c}$ for a non-negative integer $c$ and all positive $n$. Then

$$
\begin{aligned}
& \Delta^{2} \ll\|\mathbf{a}\|^{2}(\log N)^{\gamma}\left(\frac{M N^{2}}{s^{2}} \sum_{\left(D_{u}, D_{v}\right)>1} \sum_{D_{u} D_{v}} \frac{1}{D_{1}}\right. \\
&\left.+\frac{M N^{1+\delta}}{s} \sum_{s\left(D_{u}, D_{v}\right)>N^{1-\delta}} \sum_{\left[D_{u}, D_{v}\right]}+\frac{J^{2} N^{2}}{s}\right)
\end{aligned}
$$

with $\|\mathbf{a}\|^{2}=\sum\left|a_{n}\right|^{2}, \gamma=2^{c+1}+2 c-1$. 
Proof. I employ Linnik's dispersion method [11]. We write

$$
\Delta=\sum_{j} c_{j}\left(\sum_{m n \equiv u_{j}\left(\bmod D_{j} s\right)} a_{m} b_{n}-\cdots\right)
$$

with $\left|c_{j}\right| \leq 1$ and interchange the summations to obtain a representation

$$
\Delta=\sum_{m} a_{m}\left(\sum_{\substack{j \\ m n \equiv u_{j}\left(\bmod D_{j} s\right)}} c_{j} b_{n}-\sum_{j} \frac{c_{j}}{D_{j}} \sum_{\substack{m n \equiv u_{j}(\bmod s) \\\left(n, D_{j}\right)=1}} b_{n}\right) .
$$

Then $|\Delta|^{2} \leq\|\mathbf{a}\|^{2} Z$ where $Z=A-2 B+C$ with

$$
\begin{gathered}
A=\sum_{j_{1}} \sum_{j_{2}} c_{j_{1}} c_{j_{2}} \sum_{n_{1}} \sum_{n_{2}} b_{n_{1}} b_{n_{2}} \sum_{\begin{array}{c}
m n_{1} \equiv u_{j_{1}}\left(\bmod D_{j_{1}} s\right) \\
m n_{2} \equiv u_{j_{2}}\left(\bmod D_{j_{2}} s\right)
\end{array}} 1, \\
B=\sum_{j_{1}} \sum_{j_{2}} \frac{c_{j_{1}} c_{j_{2}}}{D_{j_{2}}} \sum_{n_{1}} \sum_{n_{2}} b_{n_{1}} b_{n_{2}} \sum_{\substack{m n_{1} \equiv u_{j_{1}}\left(\bmod D_{j_{1}} s\right) \\
m n_{2} \equiv u_{j_{2}}(\bmod s) \\
\left(n_{2}, D_{j_{2}}\right)=1}} 1, \\
C=\sum_{j_{1}} \sum_{j_{2}} \frac{c_{j_{1}}}{D_{j_{1}}} \frac{c_{j_{2}}}{D_{j_{2}}} \sum_{n_{1}} \sum_{n_{2}} b_{n_{1}} b_{n_{2}} \sum_{\begin{array}{l}
m n_{1} \equiv u_{j_{1}}(\bmod s) \\
m n_{2} \equiv u_{j_{2}}(\bmod s) \\
\left(n_{1}, D_{j_{1}}\right)=1,\left(n_{2}, D_{j_{2}}\right)=1
\end{array}} 1
\end{gathered}
$$

Consider $A$. The congruence condition on $m$ cannot be fulfilled unless $\left(n_{1}, D_{j_{1}} s\right)$ $=1=\left(n_{2}, D_{j_{2}} s\right)$ and $n_{2} u_{j_{1}} \equiv n_{1} u_{j_{2}}\left(\bmod s\left(D_{j_{1}}, D_{j_{2}}\right)\right)$. The innermost sum is then $M\left(2 s\left[D_{j_{1}}, D_{j_{2}}\right]\right)^{-1}+\theta$ with $|\theta| \leq 1$. Therefore,

$$
\begin{aligned}
& \left|A-\frac{M}{2 s} \sum_{\left(D_{j_{1}}, D_{j_{2}}\right)=1} \frac{c_{j_{1}} c_{j_{2}}}{D_{j_{1}} D_{j_{2}}} \sum_{\substack{u_{j_{1}} n_{2} \equiv u_{j_{2}} n_{1}(\bmod s) \\
\left(n_{1}, D_{j_{1}} s\right)=1=\left(n_{2}, D_{j_{2}} s\right)}} b_{n_{1}} b_{n_{2}}\right| \\
& \leq \frac{M}{2 s} \sum_{\sum_{\left(D_{j_{1}}, D_{j_{2}}\right)>1}} \frac{1}{\left[D_{j_{1}}, D_{j_{2}}\right]} \sum_{\substack{u_{j_{1}} n_{2} \equiv u_{j_{2}} n_{1}\left(\bmod s\left(D_{j_{1}}, D_{j_{2}}\right)\right) \\
\left(n_{1}, D_{j_{1}} s\right)=1=\left(n_{2}, D_{j_{2}} s\right)}}\left|b_{n_{1}} b_{n_{2}}\right| \\
& \quad+\sum_{j_{1}} \sum_{j_{2}} \sum_{\substack{u_{j_{1}} n_{2} \equiv u_{j_{2}} n_{1}(\bmod s) \\
\left(n_{1}, D_{j_{1}} s\right)=1=\left(n_{2}, D_{j_{1}} s\right)}} b_{n_{1}} b_{n_{2}} \mid .
\end{aligned}
$$

Similar (in fact stronger) estimates hold for $B$ and $C$ with the leading majorant replaced by

$$
\frac{M}{2 s} \sum_{\left(D_{j_{1}}, D_{j_{2}}\right)>1} \frac{1}{D_{j_{1}} D_{j_{2}}} \sum_{\substack{u_{j_{1}} n_{2} \equiv u_{j_{2}} n_{1}(\bmod s) \\\left(n_{1}, D_{j_{1}} s\right)=1=\left(n_{2}, D_{j_{2}} s\right)}}\left|b_{n_{1}} b_{n_{2}}\right| .
$$


The main terms in these estimates for $A, B$ and $C$ coincide and $Z$ does not exceed

$$
\begin{aligned}
& \frac{M}{2 s} \sum_{\left(D_{j_{1}}, D_{j_{2}}\right)>1} \frac{1}{\left[D_{j_{1}}, D_{j_{2}}\right]} \sum_{\substack{u_{j_{1}} n_{2} \equiv u_{j_{2}} n_{1}\left(\bmod s\left(D_{j_{1}}, D_{j_{2}}\right)\right) \\
\left(n_{1}, D_{j_{1}} s\right)=1=\left(n_{2}, D_{j_{2}} s\right)}}\left|b_{n_{1}} b_{n_{2}}\right| \\
& +\frac{3 M}{2 s} \sum_{\left(D_{j_{1}}, D_{j_{2}}\right)>1} \frac{1}{D_{j_{1}} D_{j_{2}}} \sum_{\substack{u_{j_{1}} n_{2} \equiv u_{j_{2}} n_{1}(\bmod s) \\
\left(n_{1}, D_{j_{1}} s\right)=1=\left(n_{2}, D_{j_{2}} s\right)}}\left|b_{n_{1}} b_{n_{2}}\right| \\
& +4 J^{2} \sum_{\substack{u_{j_{1}} n_{2} \equiv u_{j_{2}} n_{1}(\bmod s) \\
\left(n_{1} n_{2}, s\right)=1}}\left|b_{n_{1}} b_{n_{2}}\right| .
\end{aligned}
$$

The innermost sum of the first of these three bounding terms is

$$
\leq \sum_{\substack{n_{2} \leq N \\ n_{2} \equiv n_{0}(\bmod t)}}\left|b_{n_{2}}\right| \leq(\log 2 N)^{c} \sum_{\substack{n \leq N \\ n \equiv n_{0}(\bmod t)}} \tau(n)^{c},
$$

where $t=s\left(D_{j_{1}}, D_{j_{2}}\right)$ and $u_{j_{1}} n_{0} \equiv u_{j_{2}} n_{1}(\bmod t)$; recall that $\left(u_{j_{1}}, D_{j_{1}} s\right)=1$ so that $\left(u_{j}, t\right)=1$. If $t>N^{1-\delta / 2}$, then this last sum is

$$
\ll N^{\delta / 2} \sum_{n \leq N, n \equiv n_{0}(\bmod t)} 1 \ll N^{\delta / 2}\left(N t^{-1}+1\right) \ll N^{\delta} .
$$

Otherwise, Lemma 5 shows it to be $\ll N t^{-1}(\log N)^{2^{c}+c}$. Hence,

$$
\sum_{\substack{u_{j_{2}} n_{1} \equiv u_{j_{1}} n_{2}\left(\bmod s\left(D_{j_{1}}, D_{j_{2}}\right)\right) \\\left(n_{1}, D_{j_{1}} s\right)=1=\left(n_{2}, D_{j_{2}} s\right)}}\left|b_{n_{1}} b_{n_{2}}\right| \ll N(\log N)^{\gamma}\left(N t^{-1}+N^{\delta}\right)
$$

with $\gamma=2^{c+1}+2 c-1$ and where the term $N^{\delta}$ may be omitted if $t \leq N^{1-\delta / 2}$. The first term in the bound on $Z$ is thus

$$
\ll \frac{M N^{2}(\log N)^{\gamma}}{s^{2}} \sum_{\left(D_{u}, D_{v}\right)>1} \frac{1}{D_{u} D_{v}}+\frac{M N^{1+\delta}(\log N)^{\gamma}}{s} \sum_{s\left(D_{u}, D_{v}\right)>N^{1-\delta}} \frac{1}{\left[D_{u}, D_{v}\right]} .
$$

This estimate also serves for the second sum since in that case the rôle of $t$ is played by $s$, and by hypothesis $s \leq N^{1-\delta}$. The third term in the bound on $Z$ is $\ll s^{-1} J^{2} N^{2}(\log N)^{\gamma}$.

The proof of Lemma 7 is complete.

Lemma 8. The inequality of Lemma 7 remains valid if we replace $\Delta$ by

$$
\sum_{j}\left|\sum_{m n \equiv u_{j}\left(\bmod D_{j} s\right)} a_{m} b_{n}-\frac{1}{\phi\left(D_{j}\right)} \sum_{\substack{m n \equiv u_{j}(\bmod s) \\\left(m n, D_{j}\right)=1}} a_{m} b_{n}\right| .
$$


Proof. We follow the argument for Lemma 7. There is no change in the treatment of $A$, and to treat $B$ and $C$ we apply the estimate

$$
\left|\sum_{\substack{u<n \leq v \\ n \equiv r(\bmod a) \\(n, b)=1}} 1-\left(\frac{v-u}{a b}\right) \sum_{d \mid b} \mu(d)\right| \leq \sum_{d \mid b}|\mu(d)|=2^{\nu(b)},
$$

valid whenever $u \leq v$ and the positive integers $a$ and $b$ are mutually prime. Since $2^{\nu(D)} \phi(D)^{-1} \leq 2$ for every positive integer $D$, it will suffice to raise the coefficient $4 J^{2}$ to $8 J^{2}$. The only remaining (and otherwise substantial) change is the introduction into the second term bounding $Z$ of a factor $\Pi\left(1-p^{-1}\right)^{-1}$ taken over the prime divisors of $\left(D_{j_{1}}, D_{j_{1}}\right)$.

If $s\left(D_{j_{1}}, D_{j_{2}}\right) \leq N^{1-\delta / 2}, t=s\left(D_{j_{1}}, D_{j_{2}}\right)$, then again by Lemma 5 ,

$$
\sum_{\substack{n_{2} \leq N, n_{2} \equiv \bar{u}_{j_{1}} u_{j_{2}} n_{1}(\bmod s) \\ n \equiv w\left(\bmod \left(D_{j_{1}}, D_{j_{2}}\right)\right)}}\left|b_{n_{2}}\right|
$$

with $u_{j_{1}} \bar{u}_{j_{1}} \equiv 1(\bmod s)$, is $\ll N t^{-1}(\log N)^{2^{c}+c}$ uniformly for each $w$ coprime to $\left(D_{j_{1}}, D_{j_{2}}\right)$. Allowing $w$ to run over a complete set of reduced residue class representatives $\bmod \left(D_{j_{1}}, D_{j_{2}}\right)$ introduces a scaling factor $\phi\left(\left(D_{j_{1}}, D_{j_{2}}\right)\right)\left(D_{j_{1}}, D_{j_{2}}\right)^{-1}$ that cancels the above extra product. The corresponding contribution towards the modified $\Delta$ falls within the first majorant of Lemma 7 .

If $s\left(D_{j_{1}}, D_{j_{1}}\right)>N^{1-\delta / 2}$, then we omit the condition involving $w$. The corresponding contribution to $Z$ is

$$
\ll \frac{M N^{2}(\log N)^{\gamma}}{s^{2}} \sum_{s\left(D_{u}, D_{v}\right)>N^{1-\delta / 2}} \frac{1}{D_{u} D_{v}} \prod_{p \mid\left(D_{u}, D_{v}\right)}\left(1-\frac{1}{p}\right)^{-1} .
$$

Since $\phi(m) \gg m(\log m)^{-1}$ uniformly for $m \geq 2$, the reciprocal of a typical summand is

$$
\left[D_{u}, D_{v}\right] \phi\left(\left(D_{u}, D_{v}\right)\right) \gg\left[D_{u}, D_{v}\right] \phi(s)^{-1} N^{1-\delta / 2}(\log N)^{-1},
$$

and the sum is

$$
\ll \frac{M N^{1+\delta / 2}(\log N)^{\gamma+1}}{s} \sum_{s\left(D_{u}, D_{v}\right)>N^{1-\delta / 2}} \frac{1}{\left[D_{u}, D_{v}\right]},
$$

leading to a contribution towards the modified $\Delta$ that falls within the second majorant of Lemma 7.

This completes the proof of Lemma 8.

We replace the $L^{2}$ norm on a by (essentially) an $L^{\infty}$ norm. This will allow the interchange of the rôles of $M$ and $N$.

Lemma 9. Let $0<\delta<1,1 \leq s \leq N^{1-\delta}$ and assume that for some positive integer $c,\left|a_{m}\right| \leq(\tau(m) \log 2 m)^{c},\left|b_{n}\right| \leq(\tau(n) \log 2 n)^{c}$ on their respective intervals 
$(M / 2, M],(N / 2, N]$. Then

$$
\sum_{j}\left|\sum_{m n \equiv u_{j}\left(\bmod D_{j} s\right)} a_{m} b_{n}-\frac{1}{\phi\left(D_{j}\right)} \sum_{\substack{m n \equiv u_{j}(\bmod s) \\\left(m n, D_{j}\right)=1}} a_{m} b_{n}\right| \ll \lambda^{1 / 2} s^{-1} M N(\log M N)^{\gamma}
$$

with $\gamma=2^{2 c}+2 c-1$ and

$$
\lambda=\sum_{\left(D_{u}, D_{v}\right)>1} \frac{1}{D_{u} D_{v}}+\frac{s}{N^{1-\delta}} \sum_{s\left(D_{u}, D_{v}\right)>N^{1-\delta}} \frac{1}{\left[D_{u}, D_{v}\right]}+\frac{J^{2} s}{M},
$$

the implied constant depending at most upon $\delta$ and $c$.

Proof. Estimate $\|\mathbf{a}\|^{2}$ by Lemma 5.

Lemma 9 will suffice for my present purposes. The following result will help implement it.

Lemma 10. For complex numbers $a(m, j), 1 \leq j \leq J, 1 \leq m \leq x$, where $x \geq 2$,

$$
\sum_{j=1}^{J} \max _{h \leq x}\left|\sum_{m \leq h} a(m, j)\right| \ll \max _{R e(z)=(\log x)^{-1}} \sum_{j=1}^{J}\left|\sum_{m \leq x} a(m, j) m^{-z}\right|,
$$

the implied constant absolute.

Proof. Without loss of generality we may assume each inner maximum of the first sum taken at $h$ half an odd positive integer. For any $\theta>0$,

$$
\sum_{m \leq h} a(m, j)=\frac{1}{2 \pi i} \int_{\operatorname{Re}(z)=\theta} \frac{y^{z}}{z} \sum_{m \leq x} a(m, j) m^{-z} d z
$$

certainly holds if $h-1 / 4 \leq y \leq h$. Averaging over this interval we see that

$$
\sum_{m \leq h} a(m, j)=\frac{1}{2 \pi i} \int_{\operatorname{Re}(z)=\theta} K(h, z) \sum_{m \leq x} a(m, j) m^{-z} d z
$$

with

$$
K(h, z)=\frac{4}{z} \int_{h-1 / 4}^{h} y^{z} d y=\frac{4\left(h^{z+1}-(h-1 / 4)^{z+1}\right)}{z(z+1)} .
$$

In particular, $|K(h, z)| \leq \min \left(h^{\operatorname{Re} z}|z|^{-1}, h^{\operatorname{Re} z+1}(\mid z(z+1))^{-1}\right)$ uniformly in the half-plane $\operatorname{Re}(z)>0$.

The lemma follows from the fact that with $\theta=(\log x)^{-1}$,

$$
\int_{\operatorname{Re}(z)=\theta} \max _{h \leq x}|K(h, z)| d \operatorname{Im}(z) \ll \int_{|t| \leq x} \frac{d t}{\sqrt{\theta^{2}+t^{2}}}+\int_{|t|>x} \frac{x d t}{t^{2}} \ll \log x .
$$

We smooth by averaging over a short interval here, but over a long interval in Lemma 6 . 


\section{Proof of Theorem 2}

A term which falls within the bound of Theorem 2 will be called an admissible error. Let $0<\delta<1$. The Brun-Titchmarsh estimate $\psi(y, D, r) \ll y \phi(D)^{-1}$, valid uniformly for $(r, D)=1,1 \leqq D \leq y^{1-\delta}$, shows that within an admissible error we may replace the maximum over $y \leq x$ for each modulus $s D_{j}$ by the choice of half an odd integer $y=y_{j}$ in the interval $\left(x(\log x)^{-A}, x\right]$. Moreover, in $\psi\left(y_{j}, s D_{j}, r\right)$ and $\psi\left(y_{j}, s, r\right)$ we need only count those prime powers exceeding $x(\log x)^{-A}$ and coprime to $D_{j}$.

We apply Lemma 1 with $k=4$. In the notation of the remark following that lemma, with $g$ identically 1 , a typical sum over the products $n_{1} \cdots n_{r} m_{1} \cdots m_{r}$ may be expressed in the form

$$
\sum_{m_{1} \cdots m_{r} \leq x} \mu\left(m_{1}\right) \cdots \mu\left(m_{r}\right) \sum_{\substack{n_{1} \cdots n_{r} \leq x\left(m_{1} \cdots m_{r}\right)^{-1} \\ n_{1} \cdots n_{r}=b \bar{m} \bar{m}_{1} \cdots \bar{m}_{r}(\bmod D)}} \log n_{1}
$$

where $v \bar{v} \equiv 1(\bmod D)$. Lemma 6 allows us to estimate the terms for which $m_{1} \cdots$ $m_{r} \leq x^{1 / 4}$ within an error of

$$
\begin{aligned}
& \ll \sum_{m_{1} \cdots m_{r} \leq x^{1 / 4}} x\left(m_{1} \cdots m_{r}\right)^{-1}\left(\phi(D)(\log x)^{A+4}\right)^{-1} \\
& \quad+\sum_{m_{1} \cdots m_{r} \leq x^{1 / 4}} x^{1 / 2}\left(m_{1} \cdots m_{r}\right)^{-1 / 2}(\log x)^{8} \\
& \ll x \phi(D)^{-1}(\log x)^{-A}+x^{5 / 8}(\log x)^{11} .
\end{aligned}
$$

It will suffice to establish that for each $r, 1 \leq r \leq 4$,

$$
\begin{gathered}
\sum_{j=1}^{J} \mid \sum_{m_{1} \cdots m_{r} n_{1} \cdots n_{r} \equiv u_{j}\left(\bmod s D_{j}\right)} \mu\left(m_{1}\right) \cdots \mu\left(m_{r}\right) \log n_{1} \\
-\frac{1}{\phi\left(D_{j}\right)} \sum_{\substack{m_{1} \cdots m_{r} n_{1} \cdots n_{r} \equiv u_{j}(\bmod s) \\
\left(m_{1} \cdots m_{r} n_{1} \cdots n_{r}, D_{j}\right)=1}} \mu\left(m_{1}\right) \cdots \mu\left(m_{r}\right) \log n_{1} \mid
\end{gathered}
$$

is an admissible error, where each $\left(u_{j}, s D_{j}\right)=1$, every product $m_{1} \ldots m_{r}$ exceeds $x^{1 / 2}$ and $x(\log x)^{-A}<m_{1} \cdots m_{r} n_{1} \cdots n_{r} \leq y_{j}$ for the sums involving $D_{j}$.

We may dominate this sum over $j$ by $O\left((\log x)^{2 r}\right)$ similar sums with the variables $m_{u}, n_{v}$ restricted by $\frac{1}{2} M_{u}<m_{u} \leq M_{u}, \frac{1}{2} N_{v}<n_{v} \leq N_{v}$ respectively, $u=1, \ldots, r$, $v=1, \ldots, r$. We may assume that every $M_{u}$ does not exceed $x^{1 / 4}, M_{1} \cdots M_{r}>$ $x^{1 / 4}$ and that $x(\log x)^{-A}<M_{1} \cdots M_{r} N_{1} \cdots \cdots N_{r} \leq x$. According to Lemma 10, at the expense of an ultimate factor of $O\left((\log x)^{2}\right)$ and the introduction to the innermost summands of factors $\left(m_{1} \cdots m_{r}\right)^{-z_{1}-z_{2}}\left(n_{1} \cdots n_{r}\right)^{-z_{1}}$, we may ignore the three conditions on the size of the products involving the $m_{u}, n_{v}$. They are not lost since they have been transferred to the $M_{u}, N_{v}$.

We can find a subset of the $M_{u}, 1 \leq u \leq r$, whose product lies in the interval $\left[x^{1 / 4}, x^{1 / 2}\right)$. Let $M$ denote that product and collect the corresponding variables $m_{u}$ together. Collect the remaining variables amongst the $m_{u}, n_{v}$ together. We are 
reduced to estimating

$$
\sum_{j=1}^{J}\left|\sum_{m n \equiv u_{j}\left(\bmod s D_{j}\right)} a_{m} b_{n}-\frac{1}{\phi\left(D_{j}\right)} \sum_{\substack{m n \equiv u_{j}(\bmod s) \\\left(m n, D_{j}\right)=1}} a_{m} b_{n}\right|
$$

where $\left|a_{m}\right| \leq \tau_{r}(m),\left|b_{n}\right| \leq \tau_{2 r-1}(n) \log n$, and the respective sequences are supported on $\left(2^{-r} M, M\right],\left(2^{-(2 r-1)} N, N\right]$, with $x^{1 / 4} \leq M<x^{1 / 2}, x(\log x)^{-A}<M N \leq$ $x$. We apply Lemma 9 with the rôles of $N, M$ there, played by $M, M_{1} \cdots M_{r} N_{1} \cdots$ $N_{r} M^{-1}$ here. We may take $c=6$, so that $\gamma=2^{12}+11$. Since $s\left(D_{u}, D_{v}\right) \leq$ $\max s D_{j} \leq x^{1 / 4-\delta}$ is satisfied by hypothesis, the second of the sums defining $\lambda$ is empty. Moreover, in the notation of that lemma we presently have

$$
\max _{j} \phi\left(s D_{j}\right)\left(\frac{s}{M}\right)^{1 / 2} \ll x^{1 / 4-\delta}\left(\frac{s(\log x)^{A}}{x^{1 / 2}}\right)^{1 / 2} \ll x^{-\delta / 4},
$$

so that the third term in the definition of $\lambda$ gives rise to an amount

$$
\ll x^{1-\delta / 8} \sum_{j=1}^{J} \phi\left(s D_{j}\right)^{-1}
$$

in the final upper bound.

This completes the proof of Theorem 2 .

\section{Preparation for Theorem 1}

Let $\omega=\exp \left((\log \log x)^{2}\right)$. Let $\exp \left(2(\log \log x)^{6}\right)<J \leq x^{\beta}$. Express the moduli $f(j), J / 2<j \leq J$, in the form $s_{j} D_{j}$, where $s_{j}$ is made up of primes not exceeding $\omega, D_{j}$ of primes greater than $\omega$. We begin by showing that we may assume the $s_{j}$ to be comparatively small.

Lemma 11. Let $X_{j}, j=1, \ldots, h$, be independent random variables that satisfy $\left|X_{j}\right| \leq c$ almost surely. Define

$$
S_{h}=X_{1}+\cdots+X_{h}, \quad m=\sum_{j=1}^{h} \operatorname{Expect}\left(\left|X_{j}\right|\right) .
$$

Then the inequality

$$
P\left(S_{h} \geq u\right) \leq \exp \left(-\frac{u}{c}\left\{\log \frac{u}{m}-\log \log \frac{u}{m}-1\right\}\right)
$$

holds uniformly for $u \geq$ me.

Proof. This is Lemma 3.3 of [4].

Lemma 12. The number of positive integers $n$ not exceeding $z$ for which

$$
\prod_{p \mid f(n), p \leq r} p>y
$$

is

$$
\ll z \exp \left(-\frac{\log y}{50 \log r} \log \left(\frac{\log y}{\log r}\right)\right)+z^{3 / 4}
$$

uniformly for $2 \leq r \leq y \leq z$. 
Proof. Let $\rho(D)$ denote the number of solutions to $f(n) \equiv 0(\bmod D)$. Of its properties we need that it is multiplicative, $\rho\left(p^{\alpha}\right)$ in uniformly bounded, and $\rho(p) \leqq \operatorname{deg} f<p$ for $p>p_{0}$. If $\ell$ is any arithmetic function and the independent random variables $W_{p}, p_{0}<p \leq r$, are distributed according to

$$
W_{p}=\left\{\begin{array}{rll}
\ell(p) & \text { with probability } & \frac{\rho(p)}{p} \\
0 & \text { with probability } & 1-\frac{\rho(p)}{p}
\end{array}\right.
$$

then the frequency of the integers $n$ not exceeding $z$ for which $\sum \ell(p)$, taken over the prime divisors of $f(n)$ that lie in the interval $\left(p_{0}, r\right]$, belongs to $a(n y)$ set $F$ has a representation

$$
P\left(\sum_{p_{0}<p \leq r} W_{p} \in F\right)+O\left(\exp \left(-\frac{\log z}{50 \log r} \log \left(\frac{\log z}{\log r}\right)\right)\right)+O\left(z^{-1 / 4}\right),
$$

[4], Lemma 3.6. An earlier result with a weaker error is implicit in Uzdavinis, [14. Our estimate is developed from ideas of Erdös, Kac and Kubilius in Probabilistic Number Theory.

We set $\ell(p)=\log p$ on the primes, $F$ the set of reals exceeding $\frac{1}{2} \log y$. Then

$$
\sum_{p_{0}<p \leq r} \operatorname{Expect}\left(\left|W_{p}\right|\right)=\sum_{p_{0}<p \leq r} \frac{\rho(p) \log p}{p} \ll \log r,
$$

and the above probability is

$$
\ll \exp \left(-\frac{\log y}{3 \log r} \log \left(\frac{\log y}{\log r}\right)\right),
$$

by Lemma 11 with $c=\log r, u=\frac{1}{2} \log y$ if $\log y / \log r$ is suitably large, and trivially otherwise.

Lemma 12 follows rapidly.

Lemma 13. There are $\ll J \exp \left(-(\log \log x)^{2}\right)$ moduli for which

$$
s_{j}>\exp \left((\log \log x)^{6}\right) \text {. }
$$

Proof. According to Lemma 12 the number of those $j$ for which the product of all primes in $s_{j}$ exceeds $\exp \left(\frac{1}{2}(\log \log x)^{4}\right)$ falls well within the asserted bound. Moreover, if $a=2\left[(\log \log x)^{2}\right]$, then of the remaining $s_{j}$ those divisible by the ath-power of a prime are

$$
\ll \sum_{p \leq \omega} \sum_{\substack{j \leq J \\ f(j) \equiv 0\left(\bmod p^{a}\right)}} 1 \ll J \sum_{p \leq \omega} p^{-a} \ll J 2^{-a}
$$

in number.

Lemma 13 is clear.

Consider those $j$ for which $s_{j}$ has a particular value, $s$, the value not exceeding $\exp \left((\log \log x)^{6}\right)$. An application of Theorem 2 reduces to the consideration of the 
sum

$$
\sum_{t>\omega}\left(\sum_{\substack{J / 2<j \leq J \\ f(j) \equiv 0(\bmod s t)}} 1\right)^{2}
$$

Ignoring the square we obtain a doublesum that does not exceed

$$
\sum_{\substack{j \leq J \\ f(j) \equiv 0(\bmod s)}} \tau\left(s^{-1} f(j)\right) .
$$

For $s=1$ there is a long history of the treatment of such sums, beginning with van der Corput [16], who obtained a bound $\ll x(\log x)^{c}$ for a certain $c>0$. Assuming $f$ irreducible over the rationals, Erdös [6] improved $c$ to the best possible value $c=1$. His argument was modified and extended by Barban [1, Wolke [16], including the sum $\sum \tau\left(a_{n}\right)$ for a rather general sequence $a_{n}$. The treatment of Wolke would modify to give a bound $\ll s^{-1} J \log J$ for the above sum with irreducible $f$. For my present purposes it is enough to have a bound of van der Corput quality with the condition $f(j) \equiv 0(\bmod s)$ taken into account. Wolke gave a new proof of van der Corput's result, [18. The argument which follows contains another (and very short) proof of van der Corput's result. The polynomial need not be irreducible.

Lemma 14. Let $0<\delta<1$. Then

$$
\sum_{\substack{k \leq y \\ f(k) \equiv 0(\bmod s)}} \tau\left(s^{-1} f(k)\right) \ll \frac{\rho(s)}{s} y(\log y)^{\gamma}
$$

with $\left(\frac{1}{2} \gamma\right)^{\delta}=4^{\operatorname{deg} f}(\operatorname{deg} f)^{\delta}$, uniformly for $1 \leq s \leq y^{1-\delta}, y \geq 2$.

Proof. Let $0<\theta<1$. Order the prime divisors of each positive integer $n$ according to size. We may marshall those not exceeding $n^{\theta / 2}$ as a product $m_{1} \cdots m_{t}$ with at most one of the $m_{i}$ not in the interval $\left(n^{\theta / 2}, n^{\theta}\right]$. Hence, $t \leq 1+2 \theta^{-1}$. There are no more than $2 \theta^{-1}$ prime divisors of $n$ exceeding $N^{\theta / 2}$. Bearing in mind that $\tau(a b) \leq \tau(a) \tau(b)$ whether the integers $a$ and $b$ are mutually prime or not, we see that $\tau(n) \ll \tau\left(m_{i}\right)^{t}$ for some $i$, the implied constant depending at most upon $\theta$.

Choosing $\theta=\delta(\operatorname{deg} f)^{-1}$ it follows that the sum which we wish to estimate is

$$
\ll \sum_{m \ll y} \tau(m)^{t} \sum_{\substack{n \leq y \\ f(n) \equiv 0(\bmod s m)}} 1 \ll \frac{y \rho(s)}{s} \sum_{m \ll y} \frac{\tau(m)^{t} \rho(m)}{m},
$$

with the third sum

$$
\ll \prod_{p \ll y}\left(1+\frac{2^{t} \rho(p)}{p}+O\left(\frac{1}{p^{2}}\right)\right) \ll(\log y)^{2^{t} \operatorname{deg} f} .
$$

This completes the proof of Lemma 14.

A similar argument yields a proof of the first part of the Linnik-Vinogradov result in Lemma 5. The second part of that lemma may be justified as in the earliest of the references to Wolke. 


\section{Proof of Theorem 1}

For positive integers $D$, real $x \geq 2$, define

$$
E(D)=\max _{(r, D)=1} \max _{y \leq x}\left|\psi(y, D, r)-\frac{y}{\phi(D)}\right| .
$$

Application of the Brun-Titchmarsh theorem with Lemma 13 shows that the contribution to $(\Delta(J)=)$

$$
\sum_{J / 2<j \leq J} \frac{f(j)}{j} E(f(j))
$$

from the $j$ with $s_{j}>\exp \left((\log \log x)^{6}\right)$ is $\ll x \exp \left(-(\log \log x)^{2}\right)$. After Theorem 2, those of the remaining terms with $s_{j}=s$ contribute

$$
\begin{aligned}
& \ll\left(x(\log x)^{-A}+\phi(s) E(s)\right) J^{-1} \sum_{\substack{j \leq J \\
f(j) \equiv 0(\bmod s)}} 1 \\
& +x J^{-1}\left(\sum_{w<r \ll J \operatorname{deg} f}\left(\sum_{\substack{j \leq J \\
f(j) \equiv 0(\bmod s r)}} 1\right)^{2}\right)^{1 / 2}(\log x)^{4200} .
\end{aligned}
$$

Bearing in mind the constraint upon the size of $J$,

$$
\sum_{\substack{j \leq J \\ f(j) \equiv 0(\bmod s r)}} 1 \ll \rho(s r)\left(J(s r)^{-1}+1\right) \ll s^{-1} \rho(s) J \omega^{-1 / 2}
$$

uniformly for the whole range of $r$. By Lemma 14 the second of the typical bounding terms is $\ll s^{-1} \rho(s) x \omega^{-1 / 4}(\log x)^{4200+\gamma / 2}$. Altogether

$$
\Delta(J) \ll x(\log x)^{-A} \sum_{s} s^{-1} \rho(s)+\sum_{s} \rho(s) E(s)
$$

where the summations are allowed to run over (otherwise unrestricted) positive integers not exceeding $\exp \left((\log \log x)^{6}\right)$.

Results obtained by the theory of Dirichlet $L$-series (e.g. Prachar [13], IX, Satz 2.2 , cf. Satz 2.3) guarantee that $E(s) \ll s^{-1} x \exp \left(-(\log x)^{1 / 4}\right)$ uniformly for all such $s$ save possibly for the multiples of an $s_{0}$. Moreover, according to the SiegelWalfisz theorem we may assume $s_{0}$ to exceed an appropriate multiple of a chosen power of $\log x$. After another application of the Brun-Titchmarsh theorem

$$
\sum_{s} \rho(x) E(x) \ll x \exp \left(-(\log x)^{1 / 4}\right) \sum_{s \leq x} \rho(s) s^{-1}+x \sum_{t \leq x} \rho\left(t s_{0}\right)\left(t s_{0}\right)^{-1} .
$$

Since $\rho(D) \leq b^{\nu(D)}$ for some positive $b$ and all $D$, the second of these dominating terms is

$$
\ll x s_{0}^{-1} b^{\nu\left(s_{0}\right)} \sum_{t \leq x} b^{\nu(t)} t^{-1} \ll s_{0}^{-1 / 2} x(\log x)^{b} .
$$

Summing over $J=2^{-k} x^{\beta}$ with $k=0,1, \ldots$, we see that the moduli $f(D)$ in Theorem 1 with $D>\exp \left((\log \log x)^{6}\right)$ contribute $\ll x(\log x)^{-A+\operatorname{deg} f+1}$. 
We treat the complementary moduli in Theorem 1 as we did the moduli $s$ in $E(s)$, the only change being in the sum over the multiples of $s_{0}$, which becomes a sum

$$
\ll x \sum_{\substack{D \leq \exp \left((\log \log x)^{6}\right) \\ f(D) \equiv 0\left(\bmod s_{0}\right)}} D^{-1} \ll s \sum_{t=1}^{\rho\left(s_{0}\right)} \sum_{\substack{D \leq x \\ D \equiv D_{t}\left(\bmod s_{0}\right)}} D^{-1}
$$

where the $D_{t}$ comprise a set of least positive solutions to the congruence $f(D) \equiv$ $0\left(\bmod s_{0}\right)$. Typically $s_{0} \leq\left|f\left(D_{t}\right)\right| \ll D_{t}^{\operatorname{deg} f}$ and the corresponding innersum is

$$
\leq \sum_{0 \leq u \leq x}\left(D_{t}+u s_{0}\right)^{-1} \ll D_{t}^{-1}+s_{0}^{-1} \log x .
$$

The final doublesum is $\ll s_{0}^{-1 /(2 \operatorname{deg} f)} x \log x$, which suffices.

The proof of Theorem 1 is complete.

\section{WIDER UNIFORMITIES}

It is natural to approach Theorem 1 via the Large Sieve. Introducing Dirichlet characters we may dominate $\Delta(J)$ by

$$
\sum_{J / 2<j \leq J} j^{-1} \sum_{\chi \neq \chi_{0}(\bmod f(j))} \max _{y \leq x}\left|\sum_{n \leq y} \chi(n) \Lambda(n)\right| .
$$

Replacing each character by the primitive character which induces it, reduces us to the consideration of

$$
\sum_{w \ll J^{\operatorname{deg} f}} \sum_{\chi(\bmod w)}^{*} \max _{y \leq x}\left|\sum_{n \leq y} \chi(n) \Lambda(n)\right| \sum_{\substack{J / 2<j \leq J \\ f(j) \equiv 0(\bmod w)}} j^{-1} .
$$

The innersum weight is $\ll \rho(w)\left(w^{-1}+J^{-1}\right)$, a bound that for $w>J$ is poor. The sum of these weights is not more than $2 J^{-1} \sum \tau(f(j)), j \leq J$, and by a remark preceding Lemma 14 , « log $J$. Very few moduli $w$ need be considered but the standard versions of the Large Sieve are concerned more with the size than the distribution of the moduli, and naturally approach

$$
L(J)=J^{-1} \sum_{w \ll J^{\operatorname{deg} f}} \sum_{\chi(\bmod w)}^{*} \max _{y \leq x}\left|\sum_{n \leq x} \chi(n) \Lambda(n)\right|
$$

where the moduli $w$ are (otherwise) allowed to traverse all positive integers.

With $d=\operatorname{deg} f$, the proof of the Bombieri-Vinogradov theorem given by Vaughan 15] delivers a bound

$$
L(J) \ll J^{-1}\left(x+x^{5 / 6} J^{d}+x^{1 / 2} J^{2 d}\right)(\log x J)^{4} .
$$

For polynomials of degree two or more we gain a version of Theorem 1 under the restriction $6(\operatorname{deg} f-1) \beta<1$; a worse result.

Ignoring the difficulties of the reduction to bilinear forms, the Large Sieve gives for the sum considered in Lemma 8 at best a bound of the form

$$
\left(\left(M+\max D_{j}^{2}\right)\left(N+\max D_{j}^{2}\right) \sum\left|a_{m}\right|^{2} \sum\left|b_{n}\right|^{2}\right)^{1 / 2}(\log M N)^{c_{1}}, \quad c_{1}>0 .
$$


Applied to estimate $L(J)$ this will yield at best a bound

$$
\ll x(\log x)^{c_{2}}\left(M^{-1 / 2} J^{d-1}+N^{-1 / 2} J^{d-1}+x^{-1 / 2} J^{2 d-1}+J^{-1}\right)
$$

with some 'worst case' values of $M, N$ that satisfy $x(\log x)^{-A}<M N \leq x$.

We see that the case $d=1$, which arises in the Bombieri-Vinogradov theorem, is particularly well served; $M$ and $N$ need only exceed a suitable power of $\log x$, and $J$ not quite reach $x^{1 / 2}$. Once $d>1, M$ and $N$ must exceed $J^{2 d-1}$ and be appreciably large. Moreover, $M$ and $N$ should be approximately equal. Unless we take $k$ in Lemma 1 large, this is not easily arranged. Implicitly, we would need Lemma 6 for large values of $k$ with little degradation of the error term, a result that is at present beyond reach.

Disregarding all other difficulties, the best version of Theorem 1 to be reached by the application(s) of the Large Sieve indicated so far would apparently require that $(4 \operatorname{deg} f-2) \beta<1$. I show that for polynomials of degree above 8 the argument of the present paper will already achieve better.

In the notation of $\S 5$ we set $k=5$. For each non-principal Dirichlet character $(\bmod D)$, integration by parts using the Pólya-Vinogradov inequality gives

$$
L(\sigma+i t, \chi) \ll((2+|t|) \log ((2+|t|) D))^{1 / 2} D^{1 / 4}
$$

in the strip $|\sigma-1 / 2| \leq 2(\log (D(2+|t|)))^{-1}$. We can obtain a version of Lemma 4 with $r=4$ if we inflate the bound by a factor $D^{1 / 4}(T+2)^{1 / 2}(\log D(T+2))^{1 / 2}$. This legitimizes a version of Lemma 6 with $r=5$ and the second error term replaced by $x^{1 / 2} D^{1 / 4}(\log x)^{B+9}$; we employ the second of the bounds on the kernel $K(s)$ over the whole range.

In the proof proper of Theorem 2 the contribution of the terms with $m_{1} \cdots m_{4} \leq$ $x^{4 / 15}$ leads to an admissible error provided max $s D_{j} \leq x^{22 / 75}(\log x)^{-c}$ for a certain $c=c(A)>0$.

Bearing in mind that each $M_{i}$ is at most $x^{1 / 5}$, whenever $M_{1} \cdots M_{r}>x^{4 / 15}$ there is a subset of the $M_{u}$ whose product lies in $\left[x^{4 / 15}, x^{7 / 15}\right)$. We can apply Lemma 9 with (in the notation of that lemma) $M>x^{8 / 15}(\log x)^{-A}, N \geq x^{4 / 15}$.

Since $4 / 15<22 / 75$, Theorem 2 is valid for $\max s D_{j} \leq x^{4 / 15-\delta}, s \leq x^{\delta}$, and Theorem 1 for $\beta \operatorname{deg} f<4 / 15$. This last condition is more generous than $(4 \operatorname{deg} f-2) \beta<1$ as soon as $\operatorname{deg} f>8$. With even a weak improvement in Lemma 6 , the Dispersion method pulls away from the Large Sieve.

The study of the divisor function $\tau_{r}(n)$ on arithmetic progressions made by Friedlander and Iwaniec [7], using trilinear forms and Burgess' estimate for character sums, readily modifies to give a version of Lemma 6 valid for $r=6, D \leq x^{5 / 12-\varepsilon}$, and $\varepsilon>0$. This allows the choice $k=6$ in the proof of Theorem 2 and the looser restraint $\max s D_{j} \leq x^{5 / 18-\delta}$. The corresponding bound $\beta \operatorname{deg} f<5 / 18$ in Theorem 1 improves upon the best possible Large Sieve bound as soon as $\operatorname{deg} f>5$.

For cube-free moduli Friedlander and Iwaniec sharpen their result. We may correspondingly choose $k=9$ in the proof of Theorem 2, allowing max $s D_{j} \leq x^{\theta-\varepsilon}$ with $\theta=95 / 324$. The applicability of Theorem 2 towards a proof of Theorem 1 then depends upon the nature of the polynomial $f$, which may have a polynomial divisor that is already a cube.

With the simple version of the Dispersion Method employed in the present paper the natural limit of the exponents $1 / 4,4 / 15,5 / 18, \ldots, 95 / 324$ arising in the various versions of Theorem 2 appears to be $1 / 3$. 
We may further refine the Dispersion Method and the Large Sieve, too, by the introduction of Fourier Analysis on $\mathbb{R} / \mathbb{Z}$. For the Dispersion Method this devolves to the estimation of sums

$$
\sum_{J / 2<j_{1} \leq J}\left|\sum_{J / 2<j_{2} \leq J} \exp \left(2 \pi i b \overline{f\left(j_{2}\right)} / f\left(j_{1}\right)\right)\right|,
$$

possibly without the absolute values. Here $f\left(j_{2}\right) \overline{f\left(j_{2}\right)} \equiv 1\left(\bmod f\left(j_{1}\right)\right), b$ is an integer parameter and I have slightly simplified matters.

With obvious exceptions the results of Deligne yield an estimate

$$
\sum_{j \leq J} \exp (2 \pi i r(j) / p) \ll p^{1 / 2} \log p
$$

for any rational function $r(x)=f(x) / g(x), f, g$ in $(\mathbb{Z} / p \mathbb{Z})[x]$, provided $1 \leq J \leq p$ and summation is restricted to classes $j(\bmod p)$ for which $g(j)$ does not vanish. We identify $1 / g(j)$ with $\overline{g(j)}(\bmod p)$.

An elementary argument, again with some constraints upon the algebraic nature of $r(x)$, yields a similar result with the modulus $p$ replaced by a prime power $p^{n}$ and the bound by $\ll p^{n / 2} \log p^{n}$.

I have worked out the details of both the arguments. Still, we gain for the inner exponential sum over $j_{2}$ a bound that differs by only a few effectively small factors from $f\left(j_{1}\right)^{1 / 2}$. Since $f\left(j_{1}\right) \gg J^{\operatorname{deg} f}$, for polynomials of degree at least two the bound is no better than the trivial, for cubics and up much worse. A direct application of these estimates appears insufficient.

There are further possibilities but I do not consider them here. The circle of problems attached to the study of primes in short arithmetic progressions with widely spaced differences appears challenging and interesting.

\section{REFERENCES}

1. Barban, M.B. Multiplicative functions on $\sum_{R}$-equidistributed sequences, Izv. Akad. Nauk Uz. SSR (1964), 6, 13-19. MR 31:1239

2. Barban, M.B. The "large sieve" method and its applications to number theory, Uspekhi Mat. Nauk. 21 (1966), 51-102 = Russian Math. Surveys 21 (1966), 49-104. MR 33:7320

3. Bombieri, E. On the large sieve, Mathematika 12 (1965), 201-225. MR 33:5590

4. Elliott, P.D.T.A. Probabilistic Number Theory I: Mean Value Theorems, Grund. der Math. Wiss. 239, Springer Verlag, New York, Heidelberg, Berlin, 1979. MR 82h:10002a

5. Elliott, P.D.T.A. Primes, products and polynomials, preprint, to appear.

6. Erdös, P. On the sum $\sum_{k=1}^{x} d(f(k))$, J. London Math. Soc. 27 (1952), 7-15. MR 13:438f

7. Friedlander, J.R., Iwaniec, H. The divisor problem for arithmetic progressions, Acta Arithmetica 45 (1985), 273-277. MR 87b:11087

8. Gallagher, P.X. A large sieve density estimate near $\sigma=1$, Invent. Math. 11 (1970), 329-339. MR 43:4775

9. Heath-Brown, R. Sieve identities and gaps between primes, Journées Arithmétiques, Metz, 1981, Astérisque 94, Soc. Math. de France (1982), 61-65.

10. Linnik, U.V. All large numbers are sums of a prime and two squares (A problem of Hardy and Littlewood) I, Mat. Sb. (N.S.), 52 (94) (1960), 661-700. MR 22:10963

11. Linnik, U.V. The Dispersion Method in Binary Additive Problems, Leningrad, 1961, = AMS Transl. of Math. Monographs 4, Providence, Rhode Island, 1963. MR 25:3920 MR 29:5804

12. Montgomery, H.L., Topics in Multiplicative Number Theory, Lecture Notes in Math. 227, Springer-Verlag, Berlin, Heidelberg, New York, 1971. MR 49:2616

13. Prachar, K. Primzahlverteilung, Grund. der Math. Wiss. 91, Springer Verlag, Berlin, Göttingen, Heidelberg, 1957. MR 19:393b 
14. Uzdavinis, R.V. On the joint distribution of values of additive arithmetic functions of integral polynomials, Liet. TSR Mokslu Akad. darbai, Ser. B2 (18), 1959 = Trud. Akad. Nauk. Lit. SSR, Ser. B2 (18) (1960), 9-29. MR 26:100

15. Vaughan, R.C. An elementary method in prime number theory, Acta Arithmetica 37 (1980), 111-115. MR 82c:10055

16. van der Corput, J.G. "Une inégalité relative aux nombres des diviseurs", Koninklijke Nederlandsche Akademie von Wetenschapen, Proceedings, 42 (1939), 547-553. MR 1:41b

17. Wolke, D. Multiplikative Funktionen auf schnell wachsenden Folgen, J. Reine Angew. Math. 251 (1971), 54-67. MR 44:6629

18. Wolke, D. A new proof of a theorem of van der Corput, J. London Math. Soc. (2), 5 (1972), 609-612. MR 47:3336

Department of Mathematics, University of Colorado Boulder, Boulder, Colorado 80309-0395

E-mail address: pdtae@euclid.colorado.edu 